\title{
Protein - protein recognition via short amphiphilic . helices; a mutational analysis of the binding site of annexin II for p11
}

\author{
Tilmann Becker, Klaus Weber and \\ Nils Johnsson ${ }^{1}$
}

Max Planck Institute for Biophysical Chemistry, Department of Biochemistry, Am Fassberg, D-3400 Göttingen, Germany

${ }^{1}$ Present address: Massachusetts Institute of Technology, Department of Biology, Cambridge, MA 02130, USA

Communicated by K. Weber

Annexin II (p36) interacts with its ligand p11 via the short stretch of 12 amino acids (Ac-S-T-V-H-E-I-L-C-K-L-SL) situated at the $\mathrm{N}$-terminus. We have now synthesized some 37 tetradecapeptides, which differ from the original p11 binding sequence (Ac1 - 14) by single amino acid substitutions. The relative affinity of each peptide for $\mathbf{p 1 1}$ was determined by fluorescence spectroscopy using a competitive binding assay. The binding behaviour of the different peptides confirms the model of an amphiphilic $\alpha$-helix induced upon binding to p11. The apparent affinities $\Delta \Delta G_{\text {bind }}$ of the mutant peptides revealed that the $\boldsymbol{N}$-acetyl group of serine $\mathbf{1}$ and the hydrophobic side chains at positions 3, 6, 7 and 10 contribute most to the binding. The observed destabilization of the complex upon removal of single methyl groups from the hydrophobic side of the helix is comparable with the destabilization of proteins in which methyl groups have been removed from the inner core. We conclude that upon binding to p11 the hydrophobic side of the amphiphatic $\alpha$-helix becomes fully buried.

Key words: $N$-acetylation/annexins/calmodulin/EF-hand proteins/protein packing

\section{Introduction}

There is increasing evidence that the $\mathrm{Ca}^{2+}$ binding proteins with the helix-loop-helix motif (EF-hand protein family) bind to short amphiphilic helices on their target proteins. The best experimental support of this view has accumulated for calmodulin. The calmodulin binding sites on myosin light chain kinase, phosphofructokinase, phosphorylase B and the calmodulin dependent protein kinase from brain, all encompass short sequences with a high potential to form an amphiphilic $\alpha$-helix (Blumenthal et al., 1985; Lukas et al., 1986; Buschmeier et al., 1987; De Grado et al., 1987; Lin et al., 1987). We have recently proposed a similar mode of binding between p11, a member of the EF-hand protein family, and its target annexin II, formerly also called p36 or calpactin 1 heavy chain (Johnsson et al., 1988). The two proteins form in vitro and in vivo a stable tetrameric complex (Gerke and Weber, 1984, 1985a; Zokas and Glenney, 1987; Osborn et al., 1988) which due to the lack of functional EFhands in p11 (Gerke and Weber, 1985b) is not regulated by $\mathrm{Ca}^{2+}$. Annexin II belongs to a recently defined class of $\mathrm{Ca}^{2+}$ dependent membrane and lipid binding proteins (for review see Klee, 1988). p11 belongs to the S100 family of $\mathrm{Ca}^{2+}$ binding proteins, which includes beside the $\mathrm{S} 100$ proteins, calcyclin and the cystic fibrosis antigen (Gerke and Weber, 1985b; Kligman and Hilt, 1988).

The annexin II-p11 complex has a $K_{\mathrm{d}}$ of $<3 \times 10^{-8} \mathrm{M}^{-1}$ (Johnsson et al., 1988). It can be dissociated by denaturing agents or, more mildly, by certain cysteine modifying reagents (Gerke and Weber, 1985a; Johnsson and Weber, 1990). Functional and physicochemical criteria indicate that the separated polypeptides refold properly. Since annexin II is a monomer and p11 is a dimer (Gerke and Weber, 1985b) it is assumed that in the tetramer two annexin II polypeptides are attached to one p1 1 dimer. Mild proteolysis of annexin II removes the N-terminal 30 residues, often referred to as the tail domain. The resulting protease resistant core domain of the annexin molecule lacks p11 binding (Glenney et al., 1986; Johnsson et al., 1986a,b). Studies with fluorescently labelled peptides from the tail domain further delineated the p11 binding site. Strong binding is provided by the $10 \mathrm{~N}$-terminal residues and requires the $N$-acetyl group of serine residue 1 as a functional part. Circular dichroism measurements of these peptides and the arrangement of the hydrophobic residues along the sequence led us to propose that the first 12 residues form an amphiphilic helix upon binding to p11. In this model Val3, Ile6, Leu7 and Leu10 form the hydrophobic side of the helix, thought to fit into a hydrophobic cleft of the p11 molecule (Johnsson et al., 1988; see Figure 1). Interestingly the functional integrity of the $\mathrm{N}$-terminal sequence is reflected by the genomic organization of annexin II. Residues 1-15 of annexin II are separated from the rest of the coding sequences by an intron (Amiguet et al., 1990).

Here we directly evaluate the model of the annexin II-p11 interaction. We have synthesized a large number of peptides which differ from the $14 \mathrm{~N}$-terminal residues of annexin II by single amino acid substitutions. The difference in the free binding energy $\Delta \Delta G_{\text {bind }}$ of the mutant peptides allowed us to map the relative importance of each residue for the binding to 11 . The results fully confirm the model of an $\mathrm{N}$-terminal amphiphatic helix in p11 binding. Furthermore, the peptide-p11 interaction obeys the general principles proposed by Cothia and Janin (1975) for protein - protein recognition. Hydrophobicity is the major stabilizing factor of the annexin II - p11 complex and the interface between both molecules is closely packed.

\section{Results}

Rationale

Annexin II or its $\mathrm{N}$-terminal peptides can be labelled with the fluorophore acrylodan at cysteine 8 . The dye does not interfere with the binding to $\mathrm{p} 11$ and allows the association between the peptides and p11 to be monitored by fluorescence spectroscopy. Complex formation is accompanied by a shift of the maximum of the emission 
spectra from $519 \mathrm{~nm}$ to $480 \mathrm{~nm}$ and a 5-fold increase in intensity (Johnsson et al., 1988). To measure the relative affinities of the different peptides, the acrylodan labelled standard peptide Ac1 - 14 was titrated with p11 to completion and subsequently displaced by the unlabelled mutant peptides. At $50 \%$ displacement the ratio between both peptide concentrations allows the difference in free binding energy to be calculated using the formula

$$
\Delta \Delta G_{\text {aff }}=- \text { RTln } \frac{\text { [peptide] }}{\text { [Ac1-14] }} 50 \%
$$

The value $\Delta \Delta G_{\text {aff }}$, where [peptide] $50 \%$ is the free concentration of the mutant peptide necessary to obtain $50 \%$ displacement of the labelled Ac1-14, measures the difference in free binding energy $\Delta \Delta G_{\text {bind }}$ between the labelled and the mutant peptide to p11 plus the difference in free energy between the uncomplexed peptides in the solute. Assuming that the difference in free energy of the uncomplexed peptides is small compared with their differences in affinity for p11, $\Delta \Delta G_{\text {aff }}$ becomes equal to $\Delta \Delta G_{\text {bind. }}$. If not otherwise mentioned, all mutant peptides carry the $\mathrm{N}$-acetyl group and have a serine in place of the cysteine in position 8 as is the case for annexin II from chicken (Johnsson et al., 1988). Figure 1 shows the displacement of acrylodan labelled Ac1-14 by intact annexin II and the tetradecapeptide bearing a serine in position 8 . The $50 \%$ displacement at molar ratios of $1: 1$ proves that the binding site for $\mathrm{p} 11$ is fully contained in the $\mathrm{N}$-terminal 14 residues of annexin II. The change of the side chain in position 8 has no influence on the affinity for p11.

\section{A hydrophobic side chain at positions 3, 6, 7 and 10 is essential for p11 binding}

To obtain an overview of the relative importance of the different side chains we introduced in each position of the 14 residue standard peptide Ac1-14 a non-conservative substitution (Table I). Figure 2 shows that each substitution of a non-polar amino acid present on the hydrophobic side

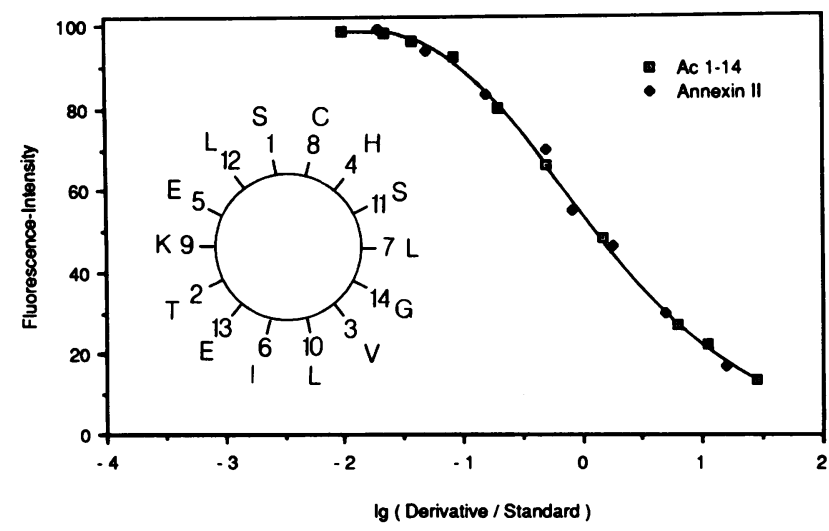

Fig. 1. Standard curve. Competitive titration of the fluorescent Ac1-14-p11 complex $(0.5 \mu \mathrm{M})$ with normal Ac1-14 or native annexin II. The fluorescence emission spectra were recorded at $20^{\circ} \mathrm{C}$ in $25 \mathrm{mM}$ imidazole $-\mathrm{HCl} \mathrm{pH} 7.3,0.1 \mathrm{M} \mathrm{NaCl}, 2 \mathrm{mM}$ EGTA, $2 \mathrm{mM}$ DTT. The emission intensity (excitation at $380 \mathrm{~nm}$ ) was integrated between 400 and $650 \mathrm{~nm}$ and plotted versus the $\log$ of the ratio of the concentration of the unlabelled derivative (unlabelled Ac1-14 or annexin II) and the labelled Ac1-14 standard peptide. Note the coincidence of the curves for the annexin molecule and the $\mathrm{N}$-terminal tetradecapeptide. The insert at the left shows the helical wheel presentation of the $\mathrm{N}$-terminal 14 residues. The hydrophobic side of the helix comprises residues 3, 6, 7 and 10 . of the helix dramatically lowers the affinity for $\mathrm{p} 11$. When valine 3 , leucine 6 or leucine 7 are individually replaced by a glutamic acid, virtually no binding occurs under our assay conditions. The mutant peptide in which leucine 10 is replaced by a glutamic acid shows an 800 -fold reduction in affinity. We do not observe comparable effects on binding when the residues on the hydrophilic side of the helix are

Table I. Tetradecapeptides usually carrying the $N$-acetyl group on residue 1

\begin{tabular}{|c|c|c|c|c|}
\hline Derivative & $\begin{array}{l}\Delta \Delta G_{\text {bind }} \\
(\mathrm{kcal} / \mathrm{mol})\end{array}$ & $\begin{array}{l}\text { Ellipticity } \\
\left.\left[\text { deg/(dmol } \mathrm{cm}^{2}\right)\right]\end{array}$ & $\begin{array}{l}\Delta \Delta G_{\mathrm{H}} \\
(\mathrm{kcal} / \mathrm{mol})\end{array}$ & $\begin{array}{l}\Delta \Delta G_{\mathrm{C}} \\
(\mathrm{kcal} / \mathrm{mol})\end{array}$ \\
\hline Ac1 - 14 & & 28600 & & \\
\hline $\mathrm{NH}_{2} 1-18$ & 4.7 & 20800 & 1.2 & 3.5 \\
\hline Ac2-14 & 4.1 & 25800 & 0.4 & 3.7 \\
\hline formyl $1-14$ & 1.8 & 28600 & 0.0 & 1.8 \\
\hline propionyl $1-14$ & 2.3 & 28550 & 0.0 & 2.3 \\
\hline S1A & 0.0 & 24200 & 0.7 & -0.7 \\
\hline SIC & 0.0 & 28500 & 0.0 & 0.0 \\
\hline S1C (acrylodan) & 0.0 & 27900 & -0.1 & 0.1 \\
\hline SIC (acid) & -0.3 & 28200 & 0.1 & -0.4 \\
\hline S1C (aminoethyl) & 0.3 & 26000 & 0.4 & -0.1 \\
\hline SIG & 1.0 & 25800 & 0.4 & 0.6 \\
\hline SIT & 0.0 & 25200 & 0.5 & -0.5 \\
\hline $\mathrm{T} 2 \mathrm{~A}$ & 1.4 & 25800 & 0.4 & 1.0 \\
\hline T2E & 2.6 & 28700 & 0.4 & 2.2 \\
\hline $\mathrm{T} 2 \mathrm{~F}$ & 1.2 & 21000 & 1.2 & 0.0 \\
\hline $\mathrm{T} 2 \mathrm{~K}$ & 1.9 & 27500 & 0.2 & 1.7 \\
\hline V3A & 3.8 & 19850 & 1.3 & 2.5 \\
\hline V3E & $>5.1$ & 29050 & -0.1 & $>5.2$ \\
\hline V3I & 2.1 & 26100 & 0.4 & 1.7 \\
\hline H4A & 1.3 & 26800 & 0.3 & 1.0 \\
\hline E5A & 0.9 & 26900 & 0.3 & 0.6 \\
\hline E5P & 3.5 & 5600 & 3.5 & 0.0 \\
\hline I6A & 2.4 & 22750 & 0.9 & 1.5 \\
\hline $\mathrm{I} 6 \mathrm{E}$ & $>4.9$ & 20150 & 1.3 & $>3.6$ \\
\hline I6V & 2.2 & 20900 & 1.2 & 1.0 \\
\hline L7A & 4.2 & 19250 & 1.4 & 2.8 \\
\hline L7E & $>5.3$ & 23300 & 0.8 & $>4.5$ \\
\hline L7F & 2.1 & 19400 & 1.4 & 0.7 \\
\hline L7V & 3.2 & 20150 & 1.3 & 1.9 \\
\hline C8S & 0.0 & 28600 & 0.0 & 0.0 \\
\hline C8P & 3.0 & 9000 & 3.0 & 0.0 \\
\hline K9E & 2.1 & 23300 & 0.8 & 1.3 \\
\hline K9R & 0.4 & 25900 & 0.4 & 0.0 \\
\hline L10E & 4.0 & 23400 & 0.8 & 3.2 \\
\hline S11D & 1.5 & 20900 & 1.2 & 0.3 \\
\hline L12K & 1.3 & 22300 & 1.0 & 0.3 \\
\hline E13K & 0.0 & 22600 & 0.9 & -0.9 \\
\hline G14L & 0.0 & 26450 & 0.3 & -0.3 \\
\hline
\end{tabular}

Mutant peptides are identified by residue position and the one letter code. The normal amino acid is given on the left and the newly introduced amino acid is on the right. Thus for instance V3E is the tetradecapeptide in which valine 3 is substituted by glutamic acid. With the mutant peptide S1C, where a cysteine replaces the normal serine 1 , further derivatives were obtained by modification of the cysteine. These involved alkylation with acrylodan, aminoethylation and the use of the cysteic acid (labelled acid in the table). Two peptides carried, instead of the $N$-acetyl group, either the $N$-formyl or the $N$-propionyl group. Peptide Ac2 - 14 lacks serine 1 and starts with the threonine carrying the $N$-acetyl group. Peptide $\mathrm{NH}_{2} 1-18$ spans the first 18 residues of the annexin II sequence but lacks the $N$-acetyl group. Acl -14 is the standard $\mathrm{N}$-acetyl tetradecapeptide serving as control. Ellipticity at $222 \mathrm{~nm}$ was measured in $80 \%$ trifluoroethanol. $\Delta \Delta G_{\text {bind }}$ values were calculated from the competitive titration curves (Figure $2-7)$ at $50 \%$ displacement of the fluorescently labelled Ac1-14 from the complex with p11. $\Delta \Delta G_{\mathrm{H}}$ and $\Delta \Delta G_{\mathrm{C}}$ values are from Figures 8 and 9 based on the assumptions given in the text. 
changed (Figure 3). Thus the introduction of either a positive or a negative charge due to chemical modification of a cysteine in position 1 (see Materials and methods) does not appreciably change the affinity of the mutant peptides. Similarly a histidine to alanine exchange at position 4 reduces the binding only 9-fold and the substitution of the glutamic acid in position 5 by alanine results in only a 4-fold lower binding affinity. A mutational influence on binding is detectable up to residue 12 . The substitution of serine 11 by aspartic acid is accompanied by a 13 -fold decrease. The exchange of the only hydrophobic residue on the hydrophilic helix side, i.e. leucine 12 , by lysine is accompanied by a 9-fold decrease in binding affinity. In contrast, the exchanges of glutamic acid 13 and glycine 14 by lysine and leucine respectively have no influence on p11 binding.

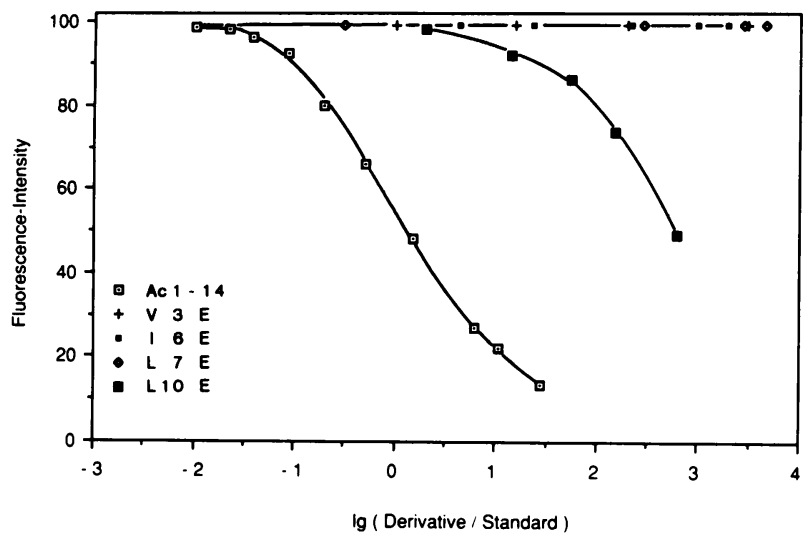

Fig. 2. Substitution of residues on the hydrophobic face of the helix by glutamic acid. Competitive titration of the fluorescent Acl-14-p11 complex $(0.5 \mu \mathrm{M})$ with different mutant peptides using the experimental conditions given in the legend to Figure 1. Mutant peptides used here and in subsequent figures are identified by residue position and the one letter code. The normal amino acid is given on the left and the newly introduced amino acid is on the right. Thus V3E is the tetradecapeptide in which Val 3 was substituted by glutamic acid. When not otherwise mentioned, all peptides contain the $\mathrm{N}$-acetyl group on residue 1 . Note the strong reduction in binding of L1OE and the virtual lack of binding for V3E, I6E and L7E.

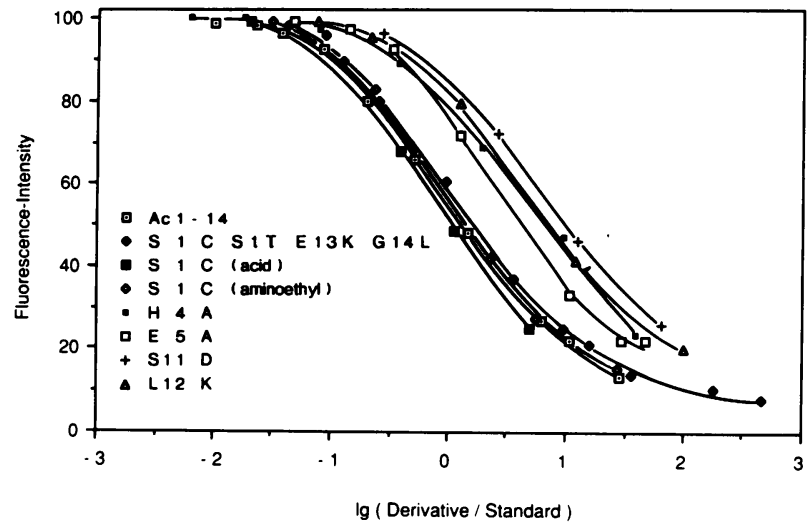

Fig. 3. Substitutions in positions 1,4 and 5 and in the C-terminal positions 11-14. Competitive titration of the fluorescent Ac1-14-p1l complex $(0.5 \mu \mathrm{M})$ with different peptides using the experimental conditions given in legend to Figure 1. The peptide nomenclature is given in Figure 2 legend. Peptide S1C with a cysteine replacing serine 1 was used to make two additional derivatives-the cysteic acid form $\left[\mathrm{S} \mathrm{C}_{(\text {acid) }}\right]$ and the aminoethylated form $\left[\mathrm{S} \mathrm{C}_{\text {(aminoethyl) }}\right]$.

\section{Positions 2 and 9}

Although positions 2 and 9 lie on the hydrophilic side of the helix, they reveal a moderate influence on the binding to 11 (Figure 4). When the charge on position 9 is changed by substitution of the lysine with a glutamic acid, binding is decreased 35-fold. It is plausible to assume that part of this decrease is due to an unfavourable charge interaction on the mutant peptide. All three glutamic acid residues of this mutant peptide (positions 5, 9 and 13) are forced to lie on the same side of the helix, once this is induced upon p11 binding. When the lysine 9 is replaced by an arginine, only a 2-fold reduction in binding is observed. The obviously rather relaxed steric requirements at position 9 support the argument that this residue is not involved in a particularly close interaction with a corresponding residue on p11 molecule.

When the threonine in position 2 is replaced by alanine or phenylalanine, the affinity for $\mathrm{p} 11$ is reduced by factors of 11 and 7 respectively (Figure 4). Interestingly, the more bulky phenyl side chain interferes less with the binding than just the deletion of the hydroxy and methyl groups in the mutant peptide containing alanine 2 . When a charge is introduced in this position, the affinity is reduced either

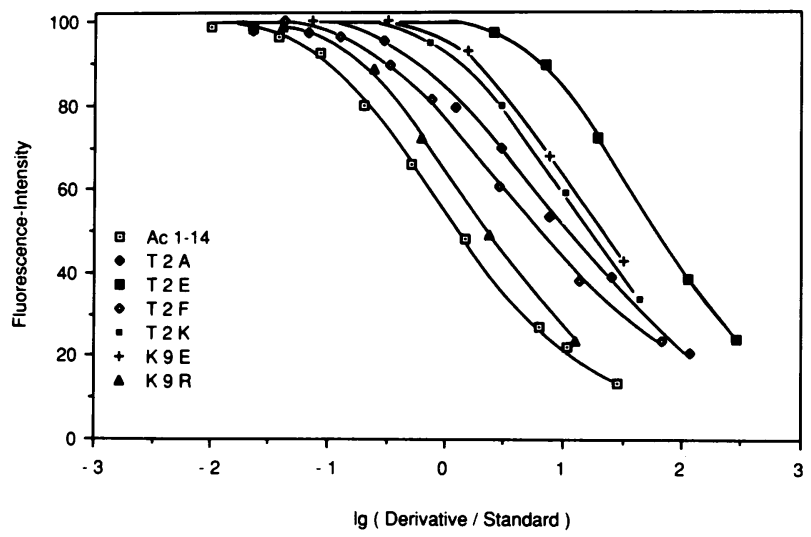

Fig. 4. Various substitutions in positions 2 and 9 of the hydrophilic side of the helix. Competitive titration of the fluorescent Acl-14-pll complex $(0.5 \mu \mathrm{M})$ with different peptides. For experimental conditions and peptide nomenclature see the legends to Figures 1 and 2 respectively.

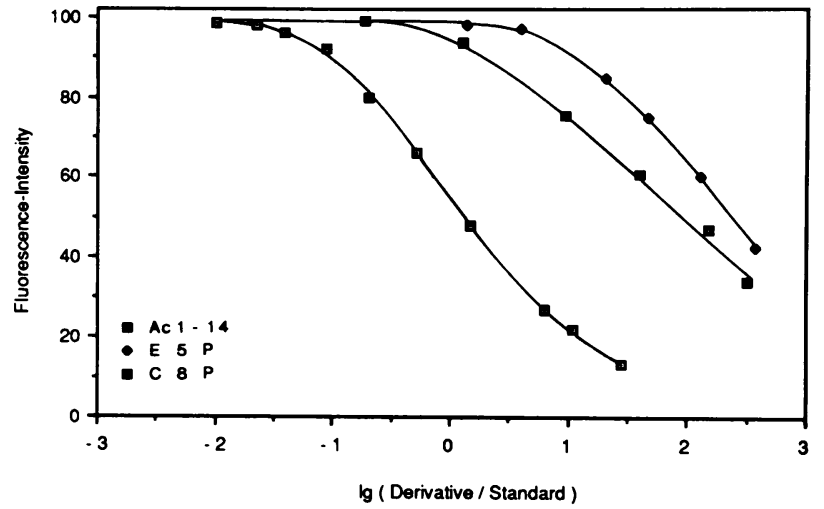

Fig. 5. Influence of helix reductions by proline substitution. Competitive titration of the fluorescent Ac1-14-p11 complex $(0.5 \mu \mathrm{M})$ with the mutant peptide containing proline at positions 5 (E5P) and 8 (C8P) respectively. Note the strong reduction in binding versus the control peptide Acl-14. For reduction of helix content see Table I. 
80-fold (glutamic acid) or 23-fold (lysine). These mutational effects at position 2 are not readily explained by the simple model and clearly require three dimensional information on the p11-peptide complex.

Testing the helix model by substitutions with proline The side chains of residues 5 (glutamic acid) and 8 (cysteine) do not directly contact the p11 interface as indicated by the fact that the introduction of an alanine at position 5 (Figure 3 ) and the chemical modification of cysteine 8 with acrylodan (Figure 1) have only a minor effect on the binding affinity. Both positions are therefore suitable to estimate the influence of the conformation of the peptide backbone on the binding to $\mathrm{p} 11$. Since a proline residue poses special requirements on the $\psi$ and $\theta$ angles of the peptide backbone, we replaced the glutamic acid in position 5 and the cysteine in position 8 each by a proline (Figure 5). The 360 -fold (proline 5) and 170-fold (proline 8) reduced affinities of the two mutant peptides seem to reflect the helix breaking tendency of the proline residue (for CD measurements see Table I). These effects support our assumption (Johnsson et al., 1988) that in all p11 binding peptides the $\alpha$-helix has to be induced to allow a strong interaction with p11 (see below).

\section{The $\mathbf{N}$-terminus}

The $N$-acetyl group of serine 1 is a functional part of the p11 binding site, since the octadecapeptide missing this posttranslational modification has an $\sim 2700$-fold reduced affinity (Johnsson et al., 1988; see also Figure 6). The 1000-fold reduced affinity of a 13 residue peptide (Ac2-14) which starts with the acetylated threonine 2 allows us to estimate the contribution of the $\mathrm{N}$-terminal imino-acetyl group

$$
\mathrm{CH}_{3}-\stackrel{\mathrm{O}}{\mathrm{C}}-\mathrm{NH}
$$

once the influence of the side chain of serine 1 is considered. This is achieved by subtracting the relative affinity of the mutant peptide with an $\mathrm{N}$-acetyl-glycine instead of the $\mathrm{N}$ -

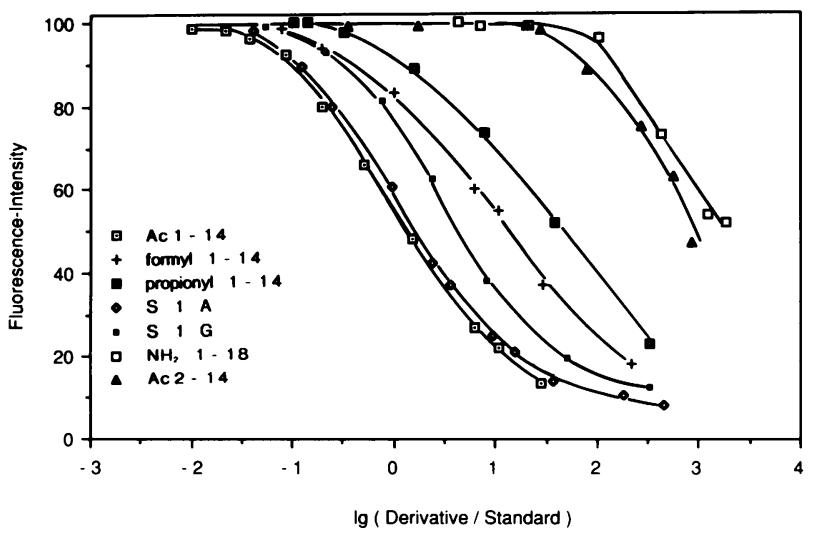

Fig. 6. Variation of the $\mathrm{N}$-terminus. Competitive titration of the fluorescent Ac1-14-p11 complex $(0.5 \mu \mathrm{M})$ with various mutant peptides. Two of the peptides carried, instead of the normal $N$-acetyl group on residue 1 , either the formyl-group (formyl 1-14) or the $N$ propionyl group (propionyl 1-14). Peptide $\mathrm{NH}_{2} 1-18$ is an 18 residue long peptide which lacks the $N$-acetyl group. Peptide Ac2-14 has a deletion of serine 1 with the $N$-acetyl group now present on the threonine, usually located in position 2 . For experimental conditions and peptide nomenclature see the legends to Figure 1 and 2 respectively. acetyl-serine in position $1(\Delta \Delta G=0.97 \mathrm{kcal} / \mathrm{mol})$ from the $\Delta \Delta G_{\text {bind }}$ of Ac2 $-14(\Delta \Delta G=4.11 \mathrm{kcal} / \mathrm{mol})$. The value of $3.14 \mathrm{kcal} / \mathrm{mol}$ for the $N$-acetyl group was further subdivided by the analysis of a peptide blocked with the formyl instead of the acetyl group. The loss of the methyl group is accompanied by a $\Delta \Delta G$ of $1.75 \mathrm{kcal} / \mathrm{mol}$. The extension of the $N$-acetyl group by a methyl group was obtained by blocking the $\mathrm{N}$-terminus of the peptide with the propionyl group. The extended propionyl peptide revealed a 48 -fold reduced affinity for $\mathrm{p} 11$. The combined results argue for a closely packed interaction between the $\mathrm{N}$-acetyl group and the interface of p11 (see also Discussion).

\section{Changes at the p11-peptide interface}

Our results predict a strong hydrophobic interaction between the non-polar side chains on the hydrophobic side of the helix and corresponding residues on the p11 molecule.The substitution by shorter but still non-polar side chains should then create cavities at the interface and consequently reduce the stability of the complexes. The effects of such mutations at positions 3,6 and 7 are shown in Figure 7. Deletion of two methyl groups at position 3 by a valine to alanine exchange reduces the free energy of binding by $3.8 \mathrm{kcal} / \mathrm{mol}$. A deletion of a single methyl group by the isoleucine to valine exchange at position 6 reduces the binding 42-fold $(\Delta \Delta G=2.2 \mathrm{kcal} / \mathrm{mol})$. Interestingly, a further deletion of two methyl groups by the introduction of an alanine adds only a $\Delta \Delta G$ of $0.2 \mathrm{kcal} / \mathrm{mol}$. While at first surprising, this result might indicate that only the $\epsilon$-methyl group of isoleucine 6 interacts with p11. The deletion of three methyl groups by the leucine to alanine exchange at position 7 causes a 1200 -fold reduction in binding. If we assume an equal distribution over the whole side chain this corresponds to a loss of $1.4 \mathrm{kcal} / \mathrm{mol} \Delta \Delta G$ per methyl group.

The interface between Ac 1-14 and p11 seems closely packed since the introduction of larger non-polar side chains decreases the stability of the complex. Thus extending the side chain of position 3 by only one methyl group due to the substitution of valine by isoleucine decreases the free energy of binding by $\sim 2.1 \mathrm{kcal} / \mathrm{mol}$ (Figure 7). A similar effect is seen when the bulky phenylalanine replaces leucine 7. However, replacement of leucine 7 by valine gives a $\Delta \Delta G_{\text {bind }}$ of $3.3 \mathrm{kcal} / \mathrm{mol}$. This exceptionally high loss of

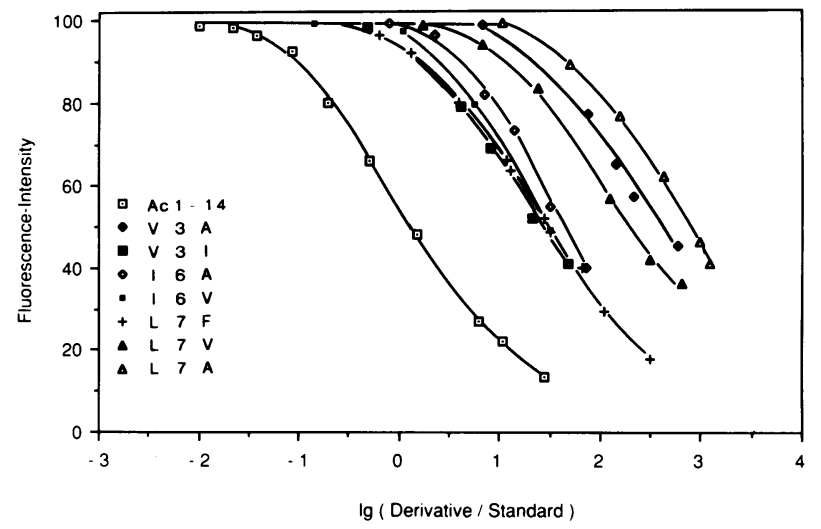

Fig. 7. Variations along the hydrophobic face of the helix. Competitive titration of the fluorescent Ac1-14-p11 complex $(0.5 \mu \mathrm{M})$ with various peptide mutants using the experimental conditions given in the legend to Figure 1. For peptide nomenclature see the legend to Figure 2. 
free binding energy per one methyl group might be caused by steric repulsion due to the differently branched methyl group of the valine side chain (Figure 7).

\section{Helix stability and complex stability}

The measured $\Delta \Delta G_{\text {bind }}$ for each peptide can be attributed to the sum of two energetic terms: the difference in helix stability $\Delta \Delta G_{\mathrm{H}}$ plus the difference in contact energy $\Delta \Delta G_{\mathrm{C}}$.

A separate evaluation of these two terms is currently not possible. To obtain a rough estimation of $\Delta \Delta G_{\mathrm{H}}$ we propose a simple calculation based on the following assumptions.

(i) The stability of the helix of the peptide in the p11-peptide complex correlates linearly with the mean residue ellipicities of the peptides at $222 \mathrm{~nm}$ in $80 \%$ trifluoroethanol/water (vol $/ \mathrm{vol}): \Delta \Delta G_{\mathrm{H}}=\mathrm{A} \times \Delta \theta_{222 \mathrm{~nm}}$.

(ii) The linear correlation is derived from those peptides where $\Delta \Delta G_{\text {bind }}$ is assumed to be solely due to a reduced stability of the helix (proline in position 5 or 8). Here: $\Delta \Delta G_{\text {bind }}=\Delta \Delta G_{\mathrm{H}}=\mathrm{A} \times \Delta \theta$.

Figure 8 shows a plot of $\Delta \Delta G_{\text {bind }}$ versus the difference in mean residue ellipticity $\Delta \theta$ of the standard peptide Ac1-14 and its mutated versions. Since residues 13 and 14 are not involved in binding (see above), their values are not considered. With the exception of two substitutions in position 1 (alanine and threonine instead of serine), all peptides show a behavior which seems consistent with our assumptions. The line in Figure 8 is defined by the origin and the values of both proline peptides, thus indicating the sole effect of conformational stability of the helix on binding. The correlation factor $A$ is $0.153 \mathrm{dcal} / \mathrm{deg} \mathrm{cm}^{2}$. The decrease in free binding energy of those mutant peptides situated on or very near this line seems only caused by a reduced helix stability, while the peptides situated on the abscissa may have only impaired contacts to p11 $\left(\Delta \Delta G_{\text {bind }}=\Delta \Delta G_{C}\right)$. The values of the peptides whose $\Delta \Delta G_{\text {bind }}$ is the sum of both terms have to lie between the abscissa and the indicated line. Here $\Delta \Delta G_{\mathrm{C}}$ can be calculated by $\Delta \Delta G_{\mathrm{C}}=\Delta \Delta G_{\text {bind }}-1.53 \times 10^{-3} \Delta \theta$. The values for all mutant peptides are listed in Table I. We stress however, that the calculation of $\Delta \Delta G_{\mathrm{C}}$ and $\Delta \Delta G_{\mathrm{H}}$ are based

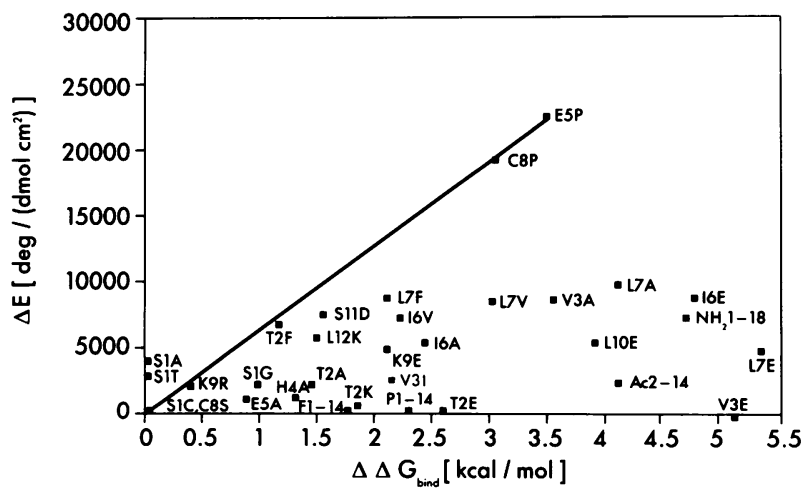

Fig. 8. Correlation of $\Delta \Delta G_{\text {bind }}$ with helix stability. Plot of the difference in helicity versus the relative binding energy $\Delta \Delta G_{\text {bind }}$. The peptide Acl-14 and all mutant peptides (Table I) except those at positions 13 and 14 are listed. The linear correlation is based on the assumption that the reduced binding affinity of the two proline derivatives (E5P and C8P) is solely due to the reduced helix stability (see Results). For helix measurements see Table I. The peptide nomenclature is given in the legend to Figure 2. on an as yet unproven assumption, which seems only to be justified by the consistent behavior of the numerous peptides in the plot of Figure 8.

\section{Discussion}

The large collection of annexin II peptides altered in a single residue allows us to weigh the importance of each residue position on the binding to $\mathrm{p} 11$. Together with the results from our proteolytic studies (Johnsson et al., 1988) we can now locate the binding site to the $12 \mathrm{~N}$-terminal residues of annexin II plus the $N$-acetyl group of serine 1 . Most of the binding energy is supplied by the side chains up to residue 10. Here the non-polar side chains of residues $3,6,7$ and 10 as well as the $N$-acetyl group contribute most to the stability of the complex. The difference in free binding energy between the various mutant peptides is sufficiently explained by our model, which proposes an amphiphilic helix covering at least residues $3-10$ of the annexin II molecule. In agreement with the model, the side chains of residues 1 , $5,8,11,12$ and 13 , which all occur on the hydrophilic side of the helix, seem to have only a moderate influence on binding. Here a significant decrease in binding is only observed by the proline substitutions, which were introduced in positions 5 and 8 respectively. The binding effects observed argue for a critical conformation of the peptide backbone at these positions and, again indirectly, for the proposed $\alpha$-helices of all p11 binding peptides.

The importance of some additional residues might have escaped our assay and could become relevant in the context of the intact annexin II molecule. A possible example could be position 14. If the helix in annexin II is not preformed but induced upon binding to $\mathrm{p} 11$, the propagation of the helix has to be stopped somewhere along the $\mathrm{N}$-terminal sequence.

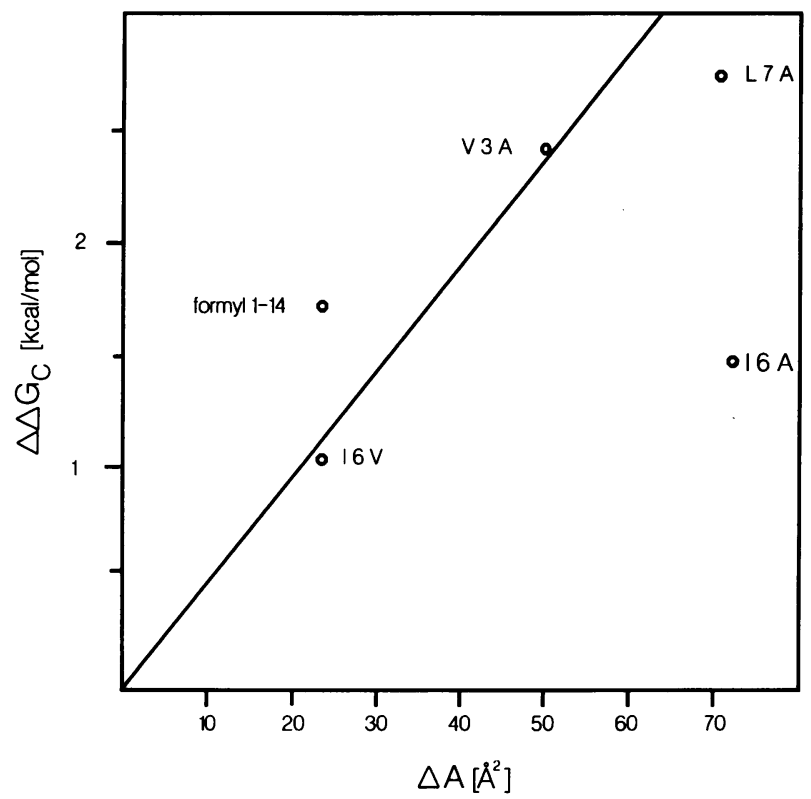

Fig. 9. Correlation of the contact energy $\Delta \Delta G_{\mathrm{C}}$ with the accessible surface area. Plot of the contact energy $\Delta \Delta G_{\mathrm{C}}$ versus the decrease in accessible surface area of those peptides where the variation is thought to cause a cavity in the hydrophobic contact area. For peptide nomenclature see the legend to Figure 2. Accessible surface areas are from Miller et al. (1987). The difference in surface area between the acetylated and formylated versions of the peptide was assumed to be equal to the difference between the Ile6 and the Val6 peptides. 
As proposed by a statistical analysis of $\alpha$-helices (Presta and Rose, 1989; Richardson and Richardson, 1989) the glycine, which occurs at position 14 of the annexin II tail, could act as an $\alpha$-helix stop signal. Indeed, the 14 residue peptide seems induced to the same amount of $\alpha$-helical structure as the longer 18 residue peptide of the annexin II molecule (our unpublished observation).

All current evidence suggests that the non-polar side chains of residues 3, 6, 7 and 10 as the hydrophobic side of the helix and the $N$-acetyl group directly contact the p11 molecule. Length reduction of these side chains without a change in their non-polar character significantly affected the binding to p11. A quantitative analysis of these data has to await a three dimensional picture of the p11-peptide complex and a more precise evaluation of each substitution on the stability of the helix in the complex $\left(\Delta \Delta G_{\mathrm{H}}\right)$. Several groups have observed a linear relationship between the free energy difference of proteins with different hydrophobic side chains in the inner core and the difference in free energy of transfer of such side chains from organic solvents to water (Kellis et al., 1989; Matsumura et al., 1988). When referred to their difference in free accessible surface area (given in $\AA^{2}$ ), a correlation of 61 and $49 \mathrm{cal} / \mathrm{mol} / \AA^{2}$ was found, depending on the exact position of the side chain in the protein (Kellis et al., 1989). In agreement with these experiments a free energy of $-50 \mathrm{cal} / \mathrm{mol}$ per $\AA^{2}$ of interacting surfaces was theoretically predicted for the association between two proteins (Cothia and Janin, 1975). The plot of $\Delta \Delta G_{\mathrm{C}}$ versus the difference in free accessible surface area of some annexin peptides is shown in Figure 9. Here only those mutant peptides are considered where the side chains on the hydrophobic side were shortened without the introduction of a different branching. In addition the $\mathrm{N}$-formylated version of the pl1 binding peptide is included. If $\Delta \Delta G_{\mathrm{C}}$ of one peptide (alanine for isoleucine 6) is neglected, a linear correlation of $48 \pm 10 \mathrm{cal} / \mathrm{mol} / \AA^{2}$ is obtained. Since the $\Delta \Delta G_{\mathrm{C}}$ values are derived by the assumption that the $\Delta \Delta G_{\mathrm{H}}$ values of each peptide correlate linearly with the ellipticity at $222 \mathrm{~nm}$ in $80 \%$ trifluoroethanol (see Results), the value of $48 \mathrm{cal} / \mathrm{mol} / \AA^{2}$ is only a rough estimate. Nevertheless this value can be used to predict that the non-polar side chains of the hydrophobic side of the $\alpha$ helix and the $N$-acetyl group at position 1 become fully buried during binding to $\mathrm{p} 11$. They seem situated in an environment comparable with that of the inner core of proteins.

A recently proposed model for the interaction of amphiphilic helices with troponin $\mathrm{C}$ and calmodulin agrees with this assumption. The hydrophobic side of these two helices fits into a hydrophobic pocket, which is a characteristic feature of both EF-hand molecules (Strynadka and James, 1990). Since p11 belongs to the same superfamily of proteins as calmodulin and troponin C, we expect a similar interaction between $\mathrm{p} 11$ and the annexin peptides. Unfortuantely not much is known about the corresponding interface of the p11 molecule. We have recently identified cysteine 82 of p11 as a residue critical in the interaction with annexin II. It is situated in the C-terminal extension, a sequence of 20 amino acids following the helix of the second helix-loop-helix motif (Johnsson and Weber, 1990). However, in contrast to annexin II, the binding site on p11 is not restricted to a short stretch of a linear sequence. Preliminary experiments with a peptide covering the Cterminal extension of p11 (residues 78-96) show only very weak interaction with annexin II (our unpublished results). Further clues about the extent and architecture of the site may have to await the results of site-specific mutagenesis and expression of the mutant p11 molecules in Escherichia coli.

\section{Materials and methods}

\section{Protein purification}

The annexin II-pll complex, formerly called protein I, was purified from porcine intestine (Gerke and Weber, 1984) with the minor modification introduced by Johnsson $e t$ al. (1988). Subunits were separated and renatured as described (Gerke and Weber, 1985a).

\section{Peptide synthesis and purity}

Peptide synthesis based on F-Moc chemistry was performed on an automatic synthesizer (Model 9050, Milligen) following the standard program of the manufacturer. To check the purity a small part of the product was treated with $95 \%$ trifluoroacetic acid to remove the protecting groups and the resin. The peptide purified by $\mathrm{C} 18$ reverse-phase HPLC was subjected to automated sequencing on an Applied Biosystems gas phase sequenator (model 470A) with an on-line PTH analyser. Alternatively a Knauer model 810 was used. When a peptide purity of $>95 \%$ was assured, the bulk preparation was subjected to treatment with acetic anhydride to acetylate the $\mathrm{N}$-terminal residue. Completion of the reaction was monitored by the Kaiser test (Kaiser et al., 1970). The acetylated peptide was removed from the resin and purified as above. Purity of peptides was analysed by amino acid analysis. Samples were hydrolysed in propionic acid/ $\mathrm{HCl}$ at $150^{\circ} \mathrm{C}$ for $1 \mathrm{~h}$. Analysis was performed on an HPLC column after pre-column derivation of the amino acids with PITC (Bidlingmeyer et al., 1984). In two cases the N-terminal residue was either $N$-formylated (Okawa and Hase, 1963) or $N$-propionylated. In these cases purity of the final product was additionally monitored by molecular weight determination using fast atom bombardment. The peptide containing cysteine in position 1 was also modified using aminoethylation with $N$-( $\beta$-iodoethyl)trifluoroacetamide (Schwartz et al., 1980), performic acid oxidation (Darbre, 1987) or reaction with acrylodan, respectively. The standard peptide Acl-14 was labelled at the single cysteine in $8 \mathrm{M}$ guanidinium- $\mathrm{HCl}$ buffered to $\mathrm{pH} 7.13$ with a 3 -fold molar excess of acrylodan at $25^{\circ} \mathrm{C}$. The reaction was stopped after $30 \mathrm{~min}$ by the addition of an excess of DTT. The labelled peptide was purified by HPLC (Johnsson et al., 1988). Purity and concentration of the final peptide stock solutions $(\sim 1-2 \mathrm{mM})$ was monitored by amino acid analysis (see above).

\section{Spectroscopic procedures}

Fluoroscence emission spectra were recorded with an SLM (Urbana, IL, USA) model 8000 spectrofluorometer with excitation at $380 \mathrm{~nm}(4 \mathrm{~nm}$ bandwidth) and the emission collected between 400 and $650 \mathrm{~nm}(4 \mathrm{~nm}$ bandwidth) as described. To obtain the exact stoichiometry of the pl1 complex with the standard peptide, the prodan labelled standard peptide Acl-14 $(1.05 \mu \mathrm{M})$ was titrated in $25 \mathrm{mM}$ imidazole $-\mathrm{HCl}, \mathrm{pH} 7.3$, $100 \mathrm{mM} \mathrm{NaCl}, 2 \mathrm{mM}$ DTT, $2 \mathrm{mM}$ EGTA with p11. At the equivalence point the fluorescence intensity reached a maximum (Johnsson et al., 1988). Subsequent addition of unlabelled peptides resulted in a decrease of the fluorescence intensity. This decrease is proportional to the degree of replacement of the labelled peptide in the p11 complex by the unlabelled peptide. The amount of unlabelled peptide necessary for a $50 \%$ replacement was used to calculate the relative binding affinity of the peptide as well as the relative binding energy $\left(\Delta \Delta G_{\text {bind }}\right)$.

CD spectra were made on a Jobin Yvon Mark V, interfaced to a microcomputer. Cuvettes of $1 \mathrm{~mm}$ path length were used. Spectra were taken in steps of $0.2 \mathrm{~nm}$ and each signal was automatically sampled and signal-averaged. Peptides were measured in the range $0-80 \%$ trifluoroethanol as described (Johnsson et al., 1988).

\section{Acknowledgements}

We thank Drs Dieter Gallwitz and Tom Jovin for access to the peptide synthesizer and the spectrofluorometer. Dr Wilhelm Kullman and HansPeter Geithe kindly provided several peptides used in this study. We appreciate the help of Uwe Plessmann in sequence analysis and thank Dr Gerard Marriott for fruitful discussions. This study was supported in part by a grant from the BMFT (Sonderforschungsbereich Grundlagen der Bioprozesstechnik; Project 5.4). 


\section{References}

Amiguet,P., D'Eustachio,P., Kristensen,T., Wetsel,R.A., Saris,C.J.M., Hunter,T., Chaplin,D.D. and Tack,B.F. (1990) Biochemistry, 29, $1226-1232$.

Bidlingmeyer,B.B., Cohen,S.A. and Tarvin,T.L. (1984) J. Chromatogr., 336, 93-104.

Blumenthal,D.K., Takio,K., Edelman,A.M., Charbonneau,H., Titani,K., Walsh,K.,A. and Krebs,E.G. (1985) Proc. Natl. Acad. Sci. USA, 82, 3187-3191.

Buschmeier,B., Meyer,H.E. and Mayr,G.W. (1987) J. Biol. Chem., 262, 9454-9462.

Cothia,C. and Janin,J. (1975) Nature, 256, 705-708.

Darbre,A. (eds) (1987) Practical Protein Chemistry-A Handbook. John Wiley \& Sons, Chichester.

De Grado,W.F., Erickson-Viipanen,F., Wolfe,H.R. and O'Neil,K.T. (1987) Proteins, 2, 20-33.

Gerke,V. and Weber,K. (1984) EMBO J., 3, 227-233.

Gerke,V. and Weber,K. (1985a) J. Biol. Chem., 260, 1688-1695.

Gerke,V. and Weber,K. (1985b) EMBO J., 4, 2917-2920.

Glenney,J.R.,Jr, Boudreau,M., Galyean,R., Hunter,T. and Tack,B. (1986) J. Biol. Chem., 261, 10485-10488.

Johnsson,N., and Weber,K. (1990) J. Biol. Chem., 265, 14464-14468.

Johnsson, N., Vandekerckhove,J., Van Damme,J. and Weber,K. (1986a) FEBS Lett., 198, 361-364.

Johnsson,N., Van,P.N., Söling,H.D. and Weber,K. (1986b) EMBO J., 5, 3455-3460.

Johnsson,N., Marriott,G. and Weber,K. (1988) EMBO J., 8, 2435-2442.

Kaiser,E., Colescott,R.L., Bossinger,L.D. and Cook,P.I. (1970) Anal. Biochem., 34, 595-598.

Kellis,J.T., Jr, Nyberg,K. and Fersht,A.R. (1989) Biochemistry, 28, $4914-4922$.

Klee,C.B. (1988) Biochemistry, 27, 6645-6653.

Kligman,D. and Hilt,D.C. (1988) Trends Biochem. Sci., 13, 437-443.

Lin,C.R., Kapiloff,M.S., Durgerian,S., Tatemoto,K., Russo,A.F. Hanson,P., Schulman,H. and Rosenfeld,M.G. (1987) Proc. Natl. Acad. Sci. USA, 84, 5962-5968.

Lukas,T.J., Burgess,W.H., Prendergast,F.G., Lau,W. and Watterson,D.M (1986) Biochemistry, 25, 1458-1464.

Matsumura,M., Becktel,W.J. and Matthews,B.W. (1988) Nature, 334, 406-410.

Miller,S., Janin,J., Lesk,A.M. and Cothia,C. (1987) J. Mol. Biol., 196, 641-656.

Okawa,K. and Hase,S. (1963) Bull. Chem. Soc. Jpn., 36, 754.

Osborn,M., Johnsson,N., Wehland,J. and Weber,K. (1988) Exp. Cell Res., $175,81-96$.

Presta,L.G.and Rose,G.D. (1988) Science, 240, 1632-1641.

Richardson,J.S. and Richardson,D.C. (1988) Science, 240, 1648-1652.

Schwartz,W.E., Smith,P.K. and Royer,G.P. (1980) Anal. Biochem., 106, $43-48$.

Strynadka,N.C.J. and James,M.N.G. (1990) Proteins, 7, 234-248.

Zokas,L. and Glenney,J.R. (1987) J. Cell. Biol., 105, 2111-2121.

Received on September 3, 1990 